\title{
ALMA: IMAGING AT THE OUTER LIMITS OF RADIO ASTRONOMY
}

\author{
Alwyn Wootten* \\ National Radio Astronomy Observatory \\ 520 Edgemont Road \\ Charlottesville, Virginia 22903-2475
}

\author{
Darrel Emerson
}

\author{
National Radio Astronomy Observatory \\ Campus Building 65 \\ 949 North Cherry Avenue \\ Tucson, AZ 85721-0655
}

\begin{abstract}
The Atacama Large Millimeter/Submillimeter Array (ALMA) is an international telescope project currently under construction in Northern Chile. Antennas arrayed over baselines up to $18 \mathrm{~km}$ in extent will constitute over $7000 \mathrm{~m}^{2}$ of collecting area, enabling ALMA to provide images of unprecedented clarity and detail. Unlike existing radioastronomical arrays, ALMA will combine interferometric and single telescope data, providing a complete range of spatial scales with complete flux recovery. Six of a planned ten planned receiver bands will be built during the construction phase; eventually ALMA will cover all atmospheric windows in the spectral wavelength range from $7 \mathrm{~mm}$ to $0.3 \mathrm{~mm}$. The combination of sensitivity, directivity, full UV coverage from the large number of individual antenna elements, precision calibration and the breadth of coverage, along with the extremely dry Chajnantor site at 16500 feet, will enable the creation of superb images of the celestial structures which emit millimeter and submillimeter photons, the most abundant photons in the Universe. Special equipment will continuously monitor atmospheric parameters, in particular the water vapor content along the telescopes' line of site, to permit real time correction of atmospheric perturbations to the observed wavefront. The observer will have a range of sophisticated data analysis techniques to be able to compensate for atmospheric and instrumental perturbations to the raw data.
\end{abstract}

Observing scripts generated at the astronomer's home institute will be transferred to the instrument, where a dynamic scheduler will match the needs of a science program to atmospheric conditions. When matched, the script is queued for observation and the data are archived. ALMA's

\footnotetext{
* The Atacama Large Millimeter Array (ALMA) is an international astronomy facility. ALMA is an equal partnership between Europe and North America, in cooperation with the Republic of Chile, and is funded in North America by the U.S. National Science Foundation (NSF) in cooperation with the National Research Council of Canada (NRC), and in Europe by the European Southern Observatory (ESO) and Spain. ALMA construction and operations are led on behalf of North America by the National Radio Astronomy Observatory (NRAO), which is managed by Associated Universities, Inc. (AUI), and on behalf of Europe by ESO.
}

average data output rate may exceed 500 GB per day. Data are then transferred to a pipeline which will produce the images; both images and data are then transmitted to regional centers for distribution to the astronomer user. The final product to the astronomer will normally consist of calibrated data cubes.

\section{INTRODUCTION}

Construction of the international Atacama Large Millimeter/Submillimeter Array project, or ALMA, has begun in radioastronomical laboratories around the world, including at the ALMA Test Facility near the Very Large Array, and in Northern Chile. The Project scope is defined in the Project Plan (ALMA Board, 2004). At project initiation, a Project Book provided a good overview of the project plans; although deprecated and no longer maintained it remains fairly complete. Wootten (2003) provided an overview of the project. Coordination of the widely distributed ALMA construction activities provides a paradigm for the coordination of signals necessary to run the array and to produce and distribute astronomical images for scrutiny by scientists around the world. In this contribution we briefly describe ALMA itself, the radiofrequency interference environment, the calibration of ALMA, and aspects of its imaging capabilities.

\section{THE ATACAMA LARGE MILLIMETER ARRAY}

\subsection{The Location and Configuration of ALMA}

ALMA will be located on the Chajnantor altiplano of the Atacama Desert of northern Chile at an elevation of $5000 \mathrm{~m}$ (16500 feet) above sea level. Its approximate coordinates are 67.75 degrees West, 23 degrees South. The site land is administered by the Chilean Ministry of National Assets and set aside by Presidential decree as a protected region for science. Measurements made in situ since 1995 confirm that the site has superior conditions for astronomical observations within its planned frequency coverage of 31.3 
to $950 \mathrm{GHz}$. Four other observatories, three of them operating at submillimeter wavelengths, currently operate on the site. The ALMA site is the highest permanent astronomical observing site in the world.

A primary safety guideline for the ALMA Project is to minimize the number of staff assigned to the $5000 \mathrm{~m}$ Array Operations Site (AOS). This guideline has many ramifications that can be summarized by the statement that ALMA will be operated remotely. That is, the array operator and all personnel involved with astronomical observations and maintenance of array instrumentation will be located at ALMA facilities at lower elevation. This leaves on the AOS only those personnel needed to assure the security of the site, people whose task it is to maintain the backend electronics and the correlator at the central electronics building on the array site, those responsible for module exchange-replacing failed instrument modules with functioning spares that are stored on the AOS-and those whose task it is to transport the antennas as needed for array reconfiguration. In order to achieve this goal the entire array must be designed and built to be modular in character, and wherever possible to be self-diagnosing. Each instrument must have provision for an adequate number of monitor points that are reported to the control computer in real time.

The ALMA antennas will be deployed in configurations populating the 216 antenna stations; the configuration will change from the compact configuration through the elongated compact configuration and the intermediate configuration's self-similar three arm spiral pattern to the most extended antenna deployment and back again in a nearly continuously reconfigured fashion. This provides changing resolution-a zoom array-where 4 antennas are moved, in normal practice, every few days by a specially designed transport vehicle. Arrays for which there is high demand may be populated for longer periods of time during the cycle.

\subsection{Recievers and Local Oscillator Distribution}

ALMA provides complete frequency coverage of all atmospheric windows from $31.3 \mathrm{GHz}$ to $950 \mathrm{GHz}$. Ten separate bands achieve this goal using an optimum choice of band edges and edge frequency ratio. Implementation of receivers covering six of these bands lies within the scope of current ALMA construction; these are those centered near $3 \mathrm{~mm}, 1.3 \mathrm{~mm}, .85 \mathrm{~mm}$ and $.45 \mathrm{~mm}$ with additional bands centered at $2 \mathrm{~mm}$ and $.6 \mathrm{~mm}$ provided by Japan. Cooled components for each receiver band are contained within assemblies known as cartridges; all lie within a vacuum chamber located in the center of the antenna receiver cabin near the reflector focus. For the cartridges covering the highest eight bands a superconducting (SIS) mixer for each of two orthogonal polarizations cooled to $4.0 \mathrm{~K}$ constitutes the initial active element.
Signals from the four IF channels travel via coaxial cables to the Downconverter Assembly in the Analog rack on one side of the receiver cabin; here the signals are mixed to 2-4 GHz baseband channels. Each polarization has four converters tuned independently through the local oscillator (LO) signal, and a switching arrangement. Accurate square law detectors are provided for continuum total power astronomy. The baseband signals follow coaxial cables to a digital rack located on the opposite side of the receiver cabin. Here each baseband channel of $2 \mathrm{GHz}$ bandwidth is digitized to 3 bits resolution at four Gigasamples per second. The four pairs of baseband channelsproduce $96 \mathrm{~Gb} / \mathrm{s}$ which is transmitted continuously from each antenna on a single optical fiber to a central building, the Array Operations Site (AOS) building for correlation.

For operations of a coherent interferometer, a phase stable reference signal, the local oscillator, has to be distributed to each antenna. ALMA generates the mm-wave or submmwave local oscillator reference signal by phase locking the difference frequency from two infrared lasers operating near $200 \mathrm{THz}$. The pair of laser signals are distributed together from the central site along line-length-corrected fiber optics to each antenna, where the beat frequency between the two infrared laser signals becomes the mm-wave LO reference.

\subsection{Correlator}

The signals from the antennas are processed by a special purpose digital signal processor, the correlator. Each channel from the antenna is requantized to two or four bits for further processing; only half the number of channels is transmitted for four bit samples. In the correlator, the digitized signals from all antennas are combined pair wise for all 2016 antenna pairs; complex correlation coefficients (fringe amplitude and phase) are produced as a function of baseline and frequency; autocorrelations are also produced for each antenna. Images of astronomical sources are created through Fourier inversion of these complex data.

The correlator throughput is $16 \mathrm{GHz}$ of signal bandwidth transmitted from the antennas in 8 basebands whose bandwidth may vary from a maximum of $2 \mathrm{GHz}$ downward in factors of two to $31.25 \mathrm{MHz}$. The smallest correlator division with all basebands active at maximum bandwidth and with all four polarization products computed would result in 64 spectral points for every baseline. For fewer baseband inputs or smaller bandwidths, additional spectral points may be provided; the maximum number of channels for narrower bandwidths is 16384 . The system clock rate is $125 \mathrm{MHz}$.

Natural time intervals associated with the correlator are $1 \mathrm{msec}$ and $16 \mathrm{msec}$. Autocorrelation results are available for time intervals as short as $1 \mathrm{msec}$ while cross-correlation results are available for intervals as short as $16 \mathrm{msec}$. If all results were to be transmitted out of the correlator for the cross-correlation products, at minimum bandwidth a 32 
GByte/s output rate would result. However, the correlator output capacity is limited to $1 \mathrm{GByte} / \mathrm{s}$, requiring some restrictions on a combination of integration time, spectral channel number, or number of baselines. Formatted data from the correlator then passes from the Technical Building at the ALMA Operations Site at which the antennas and correlator are located to the Operations Support Facility about $30 \mathrm{~km}$ away at an alititude of $2900 \mathrm{~m}$, where they are received by the Archive Processor.

\subsection{The Atacama Compact Array}

Although ALMA is sensitive to spatial frequencies from zero to that of the longest baseline in the array, that sensitivity is not uniform. To bolster sensitivity to spatial frequencies to which the main ALMA is less sensitive (between frequencies corresponding to the shortest distance between antennas (15m) and just below the $12 \mathrm{~m}$ antenna diameter), a smaller array comprised of four $12 \mathrm{~m}$ telescopes along with twelve $7 \mathrm{~m}$ antennas built to the same specifications as those in the main array will be contributed by Japan as part of its entry into the project. Data from this array (fixed in baseline except for a slight north-south extension) will be correlated in a separate correlator, from which it proceeds to the Archive Processor for inclusion in later data processing steps.

\section{RADIO FREQUENCY INTERFERENCE}

Radio Astronomy is always trying to measure natural emission at the limit of sensitivity possible with current technology. This makes it particularly susceptible to even low levels of terrestrial interference. Until now, the mm-wave part of the spectrum has been relatively free of man-made signals, but that is rapidly changing. For the ALMA array, the Chilean authorities have been very cooperative in establishing a coordination zone around the telescope; this zone is in the process of being confirmed internationally, at the ITU. At centimeter wavelengths, unwanted emission into the protected radio astronomy bands from artificial satellites has become a serious problem. Unfortunately, the mmwave spectrum is not immune from interference from satellite transmissions.

As well as ensuring protection from transmissions external to the observatory, either in space or on the ground, any radio observatory has to ensure that it's own electronics does not radiate interference. With many tens of $\mathrm{kW}$ of energy going into very high speed digital circuity, and various samplers and oscillator components at the antennas and in the central laboratory operating at frequencies from $\mathrm{MHz}$ well into the mm-wave spectrum, the adequate suppression of self-generated interference is far from trivial.

\section{ARRAY CALIBRATION}

Calibration of ALMA electronics constitutes a complex process which operates on many timescales. Here we give only the briefest overview of those calibration components most relative to ALMA's distributed nature.

\subsection{Amplitude}

The goal of amplitude and flux calibration is to convert the output voltage or counts from the correlator into brightness temperature or flux density by carefully tracking instrumental and atmospheric variations and determining accurate conversion factors. Because the adverse effects of instrumental and atmospheric variations grow rapidly with frequency, standard calibration procedures will not work well at submillimeter wavelengths. The design specifications of ALMA demand a much higher calibration accuracy than achieved by the conventional techniques used at the existing millimeter arrays, which is typically no better than $10 \%$. Producing high dynamic range $\left(>10^{3}\right)$ images, for example, requires better than a few percent accuracy on idividual interferometer baselines in amplitude calibration, and there are many scientific demands for achieving similarly high accuracy in flux calibration as well.

Absolute amplitude calibration for ALMA requires standard radio sources whose fluxes are known to $5 \%$ accuracy. Because the bright compact radio sources which are potentially useful for calibration at millimeter and submillimeter wavelengths are generally time variable, the calibration process must be something that can be repeated as often as needed. Special equipment will be needed as part of the ALMA system to provide this capability. This equipment will enable accurate antenna gain measurements followed by accurate radio source flux measurements. Variants of the standard "chopper wheel" amplitude calibration technique have been evaluated and tested. A design which incorporates several calibrated loads, one or more of which is a semi-transparent vane, is under investigation for the device to be deployed.

\subsection{Phase}

The goal of phase calibration is to measure atmospheric and instrumental delays which corrupt the incoming wave front from a celestial source in order to form the best possible image of that source. Quickly varying (compared to the timescale of one integration or calibration cycle) phase causes a loss of signal usually called 'incoherence'. Instrumental phase should be stable on timescales of many minutes; if uncorrected systematic errors will arise in the determination of the absolute visibility. Therefore calibration to measure slowly varying phase components may be achieved 
by periodic observation of astronomical point sources. Contributions to slowly varying phase include changes in the distance between the subreflector and the feed, and the stability of the LO and other electronics. A goal of the ALMA design is that its contributions to systematic error and to shorter term error, incoherence, should be well below those of the atmosphere under good observing conditions and after all available corrections have been applied.

Most of the time at Chajnantor, phase stable observations are possible only for long wavelengths or short baselines. To achieve the ALMA performance goals, compensation for atmospheric phase fluctuations will be necessary much of the time for millimeter wavelengths and modest baselines and most of the time for submillimeter wavelengths and long baselines.

Two active techniques provide correction of atmospheric errors-fast $(\sim 1.5 \mathrm{~s})$ switching of the antenna from source to a calibrator, and correction for distortions of the path by water vapor through direct observation of water in a strong atmospheric line (Water Vapor Radiometry) and subsequent phase correction at the frequency of interest. In practice, both techniques will be employed in a synergistic fashion, with fast switching providing data on the dry component of atmospheric phase, and providing calibration of the WVR, while the WVR data lessens the frequency needed for fast swtiching.

\subsubsection{Fast Switching}

Fast switching phase calibration is a technique which tracks atmospheric phase fluctuations as quickly as is reasonable by slewing the antennas to a nearby suitable calibrator source (ie, a quasar), detecting it with sufficient SNR, and then slewing back to the target source. Fast switching detects and corrects for the instrumental phase, the wet atmospheric phase, and the dry atmospheric phase. Fast switching can correct for fluctuations on time scales just under half of the cycle time. For frequencies of about $300 \mathrm{GHz}$ or less, the calibration will usually be done at the target frequency. For higher frequencies, the calibrator will be observed at $90 \mathrm{GHz}$ where the quasars are still fairly bright, and the detected phases will be scaled to the target frequency. If the calibrator and the target source are observed at different frequencies, a less frequent cross-band calibration must be employed to solve for the scaled instrumental phase drift between the two frequencies.

The details of the length of integration on the calibrator are determined by the brightness of the calibrator and the ratio of the target frequency to the calibration frequency. The overall cycle time is determined by an optimizing the overall sensitivity of the observations (i.e., a tug of war, simultaneously maximizing the time on source through long cycle times and maximizing the phase coherence through frequent calibrations and shorter cycle times). Fast switch- ing operates effectively over longer baselines; for reasons of efficiency the timescale for a fast switching cycle cannot be too short-somewhat over ten seconds is probably the fastest ALMA will switch.

\subsubsection{Water Vapor Radiometry}

Fast switching, operating over finite time periods, will not remove short term fluctuations imparted by the atmosphere. Water Vapor Radiometry (WVR) determines the amount of water vapor above each antenna radiometrically, in our case at frequencies bracketing an atmospheric water line, and calculates the phase perturbations by dead reckoning. A water vapor radiometer located at each antenna focus must be able to provide sufficient information to give a reliable estimate of the phase delay suffered by the astronomical signal arriving at the antenna. The WVR system on ALMA will operate in the $183 \mathrm{GHz}$ water line, and provide a measurement allowing correction of the atmospheric path. This system will operate to provide corrections on a timescale of about one second up to several minutes. Of course, the residual errors will include a term due to phase fluctuations not measured by the radiometer at all-'dry' air fluctuations originating in thermal variations along the path.

\section{IMAGING}

The fundamental data product of the Joint ALMA Observatory will be calibrated, deconvolved images. In addition, astronomers will receive the appropriate calibration data necessary to re-process the data. Raw data will be made available to astronomers through the ALMA archive. An online data pipeline processing system will be implemented to process science and calibration data, to support the Joint ALMA Observatory quality assurance program, and to produce the final images. The Joint ALMA Observatory will provide the off-line software necessary to re-process ALMA data, if this is needed for particular datasets. The backbone of ALMA operations will be the ALMA archive, a distributed system with nodes at the OSF, the Santiago Central Office, and ALMA Research Centers located in Europe, North America and Japan.

\section{REFERENCES}

ALMA Board 2004, ALMA Project Plan v2.0.

Baars, J. W. M. 2002 ALMA Construction Project Book Version 5.50 (http://www.alma.nrao.edu/projectbk/construction/)

Wootten, A. 2003, Proceedings of the SPIE Symposium on Astronomical Telescopes and Instrumentation, 4837, 110 . 